\title{
Reexamining Semantic Clustering: Insight from Memory Models
}

\author{
Tomoko Ishii \\ Seikei University \\ doi: http://dx.doi.org/10.7820/vli.v02.1.ishii
}

\begin{abstract}
It has been repeatedly argued that semantically related words should not be learned together because learning is impeded. However, the results of past studies are not uniform, some providing favorable results for semantic clustering, and some seem to suggest different types of similarity affect memory in different ways. The types of similarity that truly cause the problem therefore need to be examined more carefully. Focusing on visual features, which are commonly observed across different models of working memory, a study was conducted to examine if learners have difficulty memorizing a group of words that describe items with common physical features. The study compared the learning of three types of word sets: unrelated, semantically related, and physically related. While no statistically significant difference was observed between semantically related and unrelated sets, the scores for physically related sets were significantly lower than those for the other two types. This suggests the possibility that the impeding effect of semantic clustering reported in the past could be partly due to the precise nature of semantically similar words, which sometimes share visual features.
\end{abstract}

\section{Background and aim}

Among researchers of second language vocabulary learning, semantic clustering is often considered something to be avoided. This issue has long been investigated, with studies dating back to at least 50 years ago (Higa, 1963). Tinkham's (1993) was likely the study that drew the serious attention of researchers to the problem of semantically related words, with replicational studies by himself (Tinkham, 1997) and Waring (1997). They suggest that if words that fall into the same semantic field such as "fruits" (apple, orange, and pear) or "furniture" (table, chair, and bed) are learned at the same time, learning is impeded because of confusion stemming from semantic overlap. Following such research, the negative impact of semantic clustering is sometimes treated almost as if it were an established fact to the extent that the idea that grouping semantically similar words is advantageous for learning has been called a myth (Folse, 2004). However, the results of more recent research on this issue are not entirely uniform; Erten and Tekin (2008) report on the negative effect of semantic clustering, whereas Papathanasiou (2009) and Davies (2012) suggest mixed results, and Hashemi and Gowdasiaei (2005) present support for semantic clustering.

Examining this line of research, Nation and Webb (2011) point out that the effect of semantic clustering has been mostly investigated under strict experimental 
conditions. In most studies, for instance, the learners memorized words with considerable time restrictions, and the target words were presented in isolation, not in a sentence. This concern is strengthened when Hashemi and Gowdasiaei (2005, where the participants learned the words in an actual classroom setting) present favorable results for learning words in semantic sets.

Another void in the research on this issue is the lack of serious discussion on what is really causing the confusion. As a background to research in this area, researchers refer to "Interference Theory", which states that when the items to be learned have too many elements in common, the items interfere with each other and learning is impeded. However, what it means to be semantically similar is rarely discussed. Words can be connected semantically in different ways. Some are synonymous, some are in hyper- and sub-ordinate relationships. Even if the nature of the relationship is the same, there are different degrees of similarity. For instance, among coordinates of "musical instruments", many people would probably recognize piano as being closer to organ than to cymbals. It is unreasonable to assume that different types and degrees of similarity affect vocabulary learning in the same manner. Tinkham (1997) suggests that while "semantic clustering" in which the words are all from the same parts of speech has a negative impact, "thematic clustering", which includes words along one theme such as "frog" (frog, hop, slimy, pond, croak, and green) and the words are from different parts of speech, has facilitative effects. Although different labels are given to these two groupings, they are in effect both semantically connected and what Tinkham (1997) really shows is that different types of semantic relationship affect memory in different ways. More consideration on which type of similarity has a greater impact on learning is therefore necessary.

The study presented in this paper was designed as an attempt to reexamine the problem underlying semantic sets, with insight from theories of memory. Review of different models of working memory reveals that visual images are considered to be very important in memory research. The current study was conducted under the hypothesis that visual images play such an important role in human memory that they may be the central problem in learning semantically related words. The following sections will briefly review various models of working memory, followed by the report of the study.

\section{Importance of image in memory}

In the field of psychology, various models of memory have been suggested. Examining each model is well beyond the scope of this paper, but there is one commonality to the different models, which can be a clue to understanding the issue of semantic clustering. The common feature seen across various models of working memory, the first system the information goes through when it is being processed, is that they all have some form of visual component. For instance, an early model proposed by Baddely and Hitch (1974) has two initial components: the Phonological Loop, which processes sound, and the Visuo-Spatial Sketchpad, which deals with visual images. These types of information are then fed into the Central Executive, where the information is synthesized and committed to memory. When attempting to memorize something, sound and visual information are 
rehearsed in their respective components. Logie's (1995) model has a similar structure, with the Executive Functions, the Phonological Store, and the Visual Cache, all of which interact with the Knowledge Base. A more recent model, by Baddely (2000), adds the Episodic Buffer, but the Visuo-Spatial Sketchpad and the Phonological Loop still are part of his model, and the visual component remains one of the key factors in information processing.

The importance of visual image in theories of memory gives us a new perspective on research on semantic clustering. Tinkham (1997), for example, employed metal names (tin, bronze, iron, brass, lead, and steel) for one of his semantic sets, which are very difficult to differentiate visually. This is certainly an extreme case, but a fruit often has a round shape and clothes such as jacket, shirt, and coat share some physical similarity. It is not uncommon for semantically grouped words to have a similar visual image in people's mind, and this could possibly be the reason why the field has repeatedly observed the negative impact of semantic clustering. In the literature of second-language vocabulary learning, confusion between words that are similar in form, and therefore sound, has been reported (Pigada \& Schmitt, 2006). This confusion is understandable as phonology is considered to be important in many models of memory. It is then quite reasonable to hypothesize that words describing visually similar items might also cause confusion, with interference occurring within a visual component.

Given the importance attributed to imagery in information processing, as well as its possible connection to semantic clustering, an experiment was designed to see if the words for the items sharing visual features cause confusion when learned together.

\section{Study}

This study was designed under the following research question: "Does grouping semantically unrelated but physically related words have a negative impact on memory?" Three intact groups at a university in Tokyo were selected for data collection, and 64 Japanese students in either their first or second year were involved. The participants learned nonwords paired with a Japanese meaning for three different categories: "Unrelated", "Semantically related", and "Physically related." Table 1 shows the nature of each category as well as the Japanese meanings used in the study.

Each of the three above categories had three sets of six pairs, totaling 18 pairs per category, for a total number of 54 pairs to be learned. To each Japanese meaning, a nonword generated using a program named Wuggy (Keuleers \& Brysbaert, 2010) was allocated. The participants looked at six pairs displayed on a computer screen for 45 seconds and were tested on their memory immediately after this learning session (Test 1). This test asked the participants to write the Japanese meaning of each nonword. Repeating this cycle of learning and testing sessions nine times, they learned all 54 pairs and were tested on how much they could memorize. These sets were presented randomly in order to minimize the effect of order on participants' memory. 
Table 1. Nature of Categories and Japanese Meanings Prepared for the Study

\begin{tabular}{|c|c|c|}
\hline Category & Nature & Japanese meanings \\
\hline Unrelated & $\begin{array}{l}\text { There is no obvious link } \\
\text { among the words in this } \\
\text { group }\end{array}$ & $\begin{array}{l}\text { 1) Rat, cherry, clip, lotus, spoon, } \\
\text { and mountain } \\
\text { 2) Elephant, banana, tape, bur- } \\
\text { dock, kettle, and stone } \\
\text { 3) Rabbit, pear, scissors, cabbage, } \\
\text { cup, and forest }\end{array}$ \\
\hline $\begin{array}{l}\text { Semantically } \\
\text { related }\end{array}$ & $\begin{array}{l}\text { The words fall into one } \\
\text { semantic field: animals, } \\
\text { vegetables, and kitchen } \\
\text { utensils. The words were } \\
\text { selected so that they would } \\
\text { have little visual similarity }\end{array}$ & $\begin{array}{l}\text { 1) Chicken, pig, giraffe, monkey, } \\
\text { snake, and whale } \\
\text { 2) Japanese radish, cucumber, } \\
\text { spinach, okra, tomato, and egg } \\
\text { plant } \\
\text { 3) Pan, knife, cutting board, fork, } \\
\text { strainer, and ladle }\end{array}$ \\
\hline Physically related & $\begin{array}{l}\text { The words describe the } \\
\text { objects that share physical } \\
\text { features: being round, being } \\
\text { thin and long, and being } \\
\text { rectangular }\end{array}$ & $\begin{array}{l}\text { 1) Globe, watermelon, ball, pearl, } \\
\text { candy, and marble } \\
\text { 2) Pencil, fishing pole, chopsticks, } \\
\text { straw, rope, and shoe laces } \\
\text { 3) Pass card, playing card, student } \\
\text { card, business card, post card, } \\
\text { and poster }\end{array}$ \\
\hline
\end{tabular}

The participants worked on actual class activities unrelated to this study for 20 minutes and were then asked once again to write Japanese meanings for the nonwords learned earlier (Test 2). This time, all the nonwords were presented to the participants in an alphabetical order.

\section{Results}

Across all three categories, the participants memorized a large number of pairs in 45 second learning sessions (Table 2), but retention was fragile and greatly decayed with only 20 minutes of distraction (Table 3 ).

Table 2. Results of Test $1(N=64$, possible max. $=18)$

\begin{tabular}{lccccc}
\hline & Max. & Min. & Mean & SD & Std. error \\
\hline Unrelated & 18 & 1 & 13.42 & 3.93 & 0.49 \\
Semantic & 18 & 3 & 14.00 & 3.89 & 0.49 \\
Physical & 18 & 0 & 12.30 & 4.16 & 0.52 \\
\hline
\end{tabular}

Table 3. Results of Test $2(N=64$, Possible max $=18)$

\begin{tabular}{lccccc}
\hline & Max. & Min. & Mean & SD & Std. error \\
\hline Unrelated & 15 & 0 & 5.06 & 3.58 & 0.45 \\
Semantic & 16 & 0 & 5.59 & 3.91 & 0.49 \\
Physical & 16 & 0 & 4.02 & 3.50 & 0.44 \\
\hline
\end{tabular}

A repeated measures ANOVA assuming sphericity determined that mean scores for Test 1 differed statistically significantly between categories $(F(2,126)=$ 11.986, $p<0.001)$. Post hoc tests using the Bonferroni correction revealed that the 
difference in means between the unrelated and semantic sets was not statistically significant $(p=0.273)$, whereas the mean score of the physically related sets was significantly lower than the other two categories $(p=0.018$ against the unrelated sets and $p<0.001$ against the semantic sets). Likewise, a statistically significant difference was confirmed for Test $2(F(2,126)=12.069, p<0.001)$. While post hoc analysis did not show any significant difference between the unrelated and semantic sets $(p=0.336)$, the physical sets were again shown to have a significantly lower mean than the other two categories ( $p=0.007$ against the unrelated sets, and $p<$ 0.001 against the semantically similar sets). Partial eta-squared for these analyses were 0.306 for Test 1 and 0.286 for Test 2 .

In addition to the mean scores, it is worth noting the frequency of cases where participants confused words from the same set. For instance, in Test 2, several participants answered "pass card (teiki-ken)" when the correct answer would be "student card (gakusei-sho)". Those two words were presented together in the learning session, and such cases indicate confusion in memory across word pairs they tried to memorize at the same time. Among unrelated sets, there was only one such case, while semantically related sets had 13 cases. In contrast, physically related sets observed as many as 36 cases of such confusion. The number of occurrences being limited as a whole, it was not possible to run a statistical analysis on this, but the difference is considerable. It should be noted however that there were 33 participants who did not show any confusion in any of the conditions. Most of these participants scored very low in Test 1 , meaning they remembered few words and so there were fewer opportunities for confusion. However, two participants were able to recall over 40 out of 54 words after 20 minutes of distraction with no instances of confusion. There was thus a large degree of individual difference in the occurrence of such confusion.

\section{Discussion and conclusions}

The results presented above suggest that it is harder to learn physically related words at the same time than learning unrelated or semantically related words, although variation among individuals needs to be recognized. The difficulty may stem from the confusion generated by processing similar visual images of items described by the words, as the visual component is considered important in theories of working memory. This study did not observe any advantage or disadvantage of the semantic sets that avoided visual similarity of the items described, which suggests that the impeding effect of such clustering reported in the past could be explained partly by the nature of semantically related words, in that they sometimes share visual features.

It should be noted, however, that the results presented in this paper could possibly be an artifact of the selection of Japanese concepts as well as that of nonwords. It is hard to argue against the critique that what is considered as visual similarity is determined by the rather subjective judgment of the author. Furthermore, this study was conducted under an experimental setting, not in actual classrooms, and does not examine any long-term effect. It is therefore necessary that the research be replicated with different meanings and nonwords, as well as in different contexts. 
Visual similarity is an aspect of memory that has not been well addressed in the literature of semantic clustering. In this small-scale study the negative impact of visually related but semantically unrelated sets were observed, while similar effects for semantically related but visually unrelated sets was not observed. These results question the often-cited source of difficulty in learning semantically grouped words. With further study employing different word meanings, as well as different types of visual features, the real source of confusion caused by semantic clustering could be further clarified.

\section{References}

Baddely, A.D. (2000). The episodic buffer: A new component of working memory? Trends in Cognitive Sciences, 4 (11), 417-423. doi:10.1016/S1364-6613(00) 01538-2

Baddely, A.D., \& Hitch, G.J. (1974). Working memory. In G.A. Bower (Ed.), Recent advances in learning and motivation (Vol. 8, pp. 47-89). New York: Academic Press.

Davies, M.J. (2012). A comparison of the memorization and recall of vocabulary in semantic and thematic groups. Vocabulary Education \& Research Bulletin, 1 (1), 3-4. http://jaltvocab.weebly.com/uploads/3/3/4/0/3340830/verb-vol1.1.pdf

Erten, I.H., \& Tekin, M. (2008). Effects on vocabulary acquisition of presenting new words in semantic sets versus semantically-unrelated sets. System, 36 (3), 407-422. doi:10.1016/j.system.2008.02.005

Folse, K.S. (2004). Vocabulary myths. Michigan: The University of Michigan Press.

Hashemi, M.R., \& Gowdasiaei, F. (2005). An attribute-treatment interaction study: Lexical-set versus semantically-unrelated vocabulary instruction. RELC Journal, 36 (3), 341-361. doi:10.1177/0033688205060054

Higa, M. (1963). Interference effects of intralist word relationships in verbal learning. Journal of Verbal Learning and Verbal Behavior, 2, 170-175. doi:10. 1016/S0022-5371(63)80082-1

Keuleers, E., \& Brysbaert, M. (2010). Wuggy: A multilingual pseudoword generator. Behavior Research Methods, 42 (3), 627-633. doi:10.3758/BRM.42. 3.627

Logie, R.H. (1995). Visuo-spatial working memory. Hove: Lawrence Erlbaum Associates.

Nation, I.S.P., \& Webb, S. (2011). Researching and analyzing vocabulary. Boston: Heinle Cengage Learning.

Papathanasiou, E. (2009). An investigation of ways of presenting vocabulary. ELT Journal, 63 (2), 313-322. doi:10.1093/elt/ccp014

Pigada, M., \& Schmitt, N. (2006). Vocabulary acquisition from extensive reading: A case study. Reading in a Foreign Language, 18 (1), 1-27. Retrieved from http:// nflrc.hawaii.edu/rfl/april2006/pigada/pigada.pdf 
Tinkham, T.M. (1993). The effect of semantic clustering on the learning of second language vocabulary. System, 21, 371-380. doi:10.1016/0346-251X(93) 90027-E

Tinkham, T. (1997). The effects of semantic and thematic clustering on the learning of second language vocabulary. Second Language Research, 13 (2), 138-163. doi:10.1191/026765897672376469

Waring, R. (1997). The negative effects of learning words in semantic sets: A replication. System, 25, 261-274. doi:10.1016/S0346-251X(97)00013-4 